\title{
A POWER-SAVING ALGORITHM AND A POWER-AWARE ROUTING SCHEME FOR IEEE 802.11 AD HOC NETWORKS
}

\author{
Nikos Pogkas ${ }^{1}$ and George Papadopoulos ${ }^{2}$ \\ ${ }^{\prime}$ Department of Electrical and Computer Engineering University of Patras, Campus of Rio, \\ Greece E-mail: npogas@ee.upatras.gr; ${ }^{2}$ Industrial Systems Institute, Rion Patras Greece
}

\begin{abstract}
This paper proposes a local power saving algorithm combined with a power-aware routing scheme to provide energy-efficient operation of the network. The power saving algorithm reduces communication energy consumption whereas the power aware routing scheme increases node lifetime. Another objective of the proposed routing strategy is the selection of stable paths in order to achieve robust network operation; to accomplish this scope a new routing metric is presented combining the residual energy level of nodes with an estimation of the stability of links. Simulation studies indicate a reduction in energy consumption and a significant increase in node lifetime whereas the network performance (delivery ratio and routing overhead) is not affected significantly.
\end{abstract}

Key words: Power aware routing, power saving mode, low power mode,

\section{INTRODUCTION}

In recent years there has been increased research interest in the area of ad-hoc networks, which can operate without the presence of any preinstalled network infrastructure, have distributed robust operation and can adapt well in network dynamics such as mobility of hosts or topological changes. These network properties are desirable in many situations such as military, law enforcement or disaster relief operations.

This work is based on the design and implementation of a low cost ad-hoc network for disaster relief operations ${ }^{1}$. Our application objective was a search and rescue operation ${ }^{2}$ after an earthquake in a collapsed apartment, building or house. In each apartment or building there should be a number of small preinstalled, low-cost, battery operated autonomous devices (sensor nodes) equipped with a camera CMOS sensor, an audio microphone and a wireless interface. The rescue teams using mobile central units, roaming over the collapsed building should 
collect captured images and recorded audio from the remote sensor nodes inside the collapsed building in order to find trapped people and rescue them.

This work focuses on routing and network energy consumption issues. A combined efficient way for dealing with idle time (low power mode) and communication (power aware routing) is proposed for a powerful network energy management solution. Although this work is based on $\mathrm{DSR}^{3}$ and IEEE $802.11 \mathrm{~b}$, the principles apply to other routing and MAC protocols.

\section{LOW POWER MODE ALGORITHM}

Since the mobile nodes of an ad hoc network operate using batteries, it is important to minimize the power consumption of the network. Existing network solutions that improve energy efficiency focus on: transmit power control, low power mode algorithms and power aware routing algorithms.

While transmit power control increases the network capacity and reduces interference, its use in reducing energy consumption is based heavily in the hardware specifications of the wireless interface ${ }^{4}$. The use of multi-hop paths saves energy only when the path attenuation dominates the static energy consumption of the hardware, a case that occurs less frequently than is typically believed. As a result our energy efficient solution is based on the combination of a low power mode algorithm and a power aware routing strategy, presented in the next sections.

The basic idea of the proposed Low Power Mode algorithm (LPM) is to reduce the idle power consumption by turning off the radios of nodes that are idle. The algorithm's operation is driven entirely by the communication in the network. The main design considerations of our LPM implementation are the following: distributed robust operation in ad hoc networks, the use of the algorithm should not affect significantly network performance, no need for synchronization between the nodes (the synchronization of nodes proposed in various $\mathrm{Chen}^{5}$ is difficult to implement in dynamic topologies and induces a significant network overhead), no periodic exchange of packets or beacons (periodic transmission of packets or beacons proposed by Zheng ${ }^{6}$ increases the energy consumption unnecessarily when the network is idle, also channel bandwidth and network capacity is decreased while the beacons or control packets contend for channel access with normal data packets), low implementation complexity and no need for modifications at the MAC layer (in order to be easily deployed with current commercial and future wireless products). 


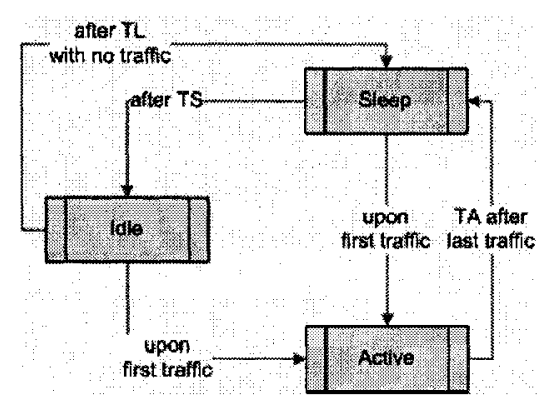

Figure 1. State diagram of the LPM algorithm

The algorithm has been implemented as an intermediate network driver at the LLC OSI layer for commercial IEEE 802.11 wireless cards. In Fig. 1 we present the state diagram of the algorithm. When a node is idle it periodically turns off its radio for duration $T s$ entering the Sleep state. After $T_{S}$ it enters the Idle state for a period of $T_{L}$ where it listens for incoming packets. When it receives a broadcast or unicast packet addressed to that node it enters the Active state where it remains for duration of $T_{A}$. The same state transition occurs when there is a packet available at the egress queue ready for transmission. When a node is in the Sleep state it cannot receive any packets from any other node. As a result, a mechanism must be implemented in order to guarantee the successful reception of packets for a node that periodically enters the Sleep sate. This mechanism is presented in Fig. 2. Every node that transmits a packet remains in the Active state for $T_{A}$. If the destination is a node that is possibly in the power saving state (namely transits periodically between the Idle and the Sleep state), the source node must retransmit the packet $R$ times with an interval $T_{O}$ in order to overlap the packet transmission with the destination's Idle state.

In order to reduce possible local congestion or overhead in the network the following mechanism has been implemented. Each node maintains a table (Next-hop Nodes Table) with the possible states of its next-hop neighbours in order to decide if it has to make the $R$ retransmissions or not. If the node transmits or receives a packet from a neighbour node, it marks this node as active in its table. After $T_{A}$ seconds it marks the same node as possible-inactive (power-saving state) until it transmits successfully or receives another packet from that node. If the wireless interface is working in promiscuous mode, the nodes can trace any packet transmission in the network in order to update the status of next-hop nodes, improving the algorithm's performance. 


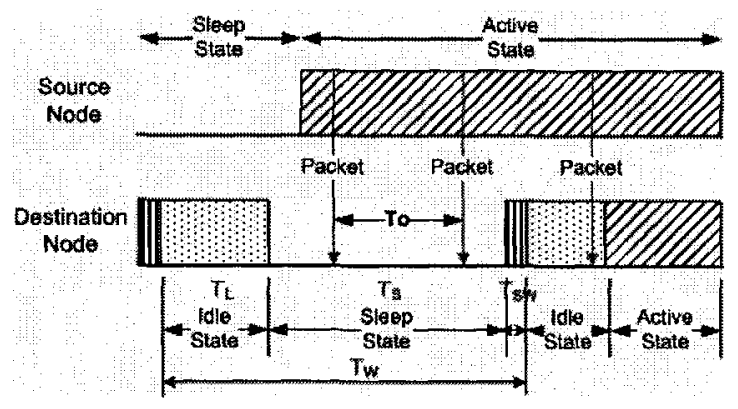

Figure 2. Packet retransmission mechanism

The information regarding the active state of a destination node in the next-hop table is accurate enough to coordinate transmissions since the source node has detected a successful transmission or reception of a packet from that node based on a MAC layer acknowledgement. If the destination node is in the power saving state then the source node will initiate the retransmission mechanism. If the destination node enters the active state the source node will receive an acknowledgement and will stall any pending retransmissions of the packet.

However, during a broadcast transmission there is no MAC-ACK, so the source node has to transmit a broadcast packet every $T_{O}$ seconds for $R$ times. In order to reduce this overhead, a variable is maintained ( $T$ _last_broadcast) which contains the last time that a broadcast packet has been transmitted. Broadcast packets will cause every next-hop node to enter the active state for at least a period of $T_{A}$. Thus, when a sending node transmits a broadcast packet at time $t<T_{A}+T_{-}$last_broadcast it assumes that all its next-hop nodes are still in the active state and the packet is transmitted normally without any retransmissions. This mechanism reduces significantly the number of retransmissions in the network which are caused by the use of the algorithm.

In the presence of unstable links or high mobility, the above mechanism performs sufficiently well and in some cases improves network performance as a result of the additional retransmissions imposed in unicast and broadcast packets. The major disadvantages of the proposed LPM algorithm are the increased latency during a route discovery (a basic problem found in most of the asynchronous low power mode algorithms) and the additional overhead induced by broadcast packets that are transmitted in the network (and especially for packets that are flooded in the entire network).

In the next paragraphs we present a systematic approach to address the LPM algorithm's design parameters and we calculate the maximum achieved energy efficiency of such an algorithm. An idle node not implementing the LPM algorithm in a time period $T_{W}=T_{L}+T_{S}+T_{S W}$ will consume energy 


$$
E_{1}=P_{L} * T_{L}+P_{L} * T_{S}+P_{L} * T_{S W}
$$

During the same period an idle node that implements the algorithm will consume energy

$$
E_{2}=P_{L} * T_{L}+P_{S}^{*} T_{S}+P_{S W} * T_{S W}
$$

where $P_{L}$ is the power consumed at the idle state, $P_{S}$ is the power consumed when the radio is off, $T s$ is the time a node spends in the sleeping state and $T_{L}$ in the listening state. $T s w$ is the time needed to switch the radio from off to on plus the time needed to switch from on to off and, during this period, the power consumption is approximately equal to $P_{L}\left(P_{S W} \approx P_{L}\right)$, as measured in many experimental scenarios ${ }^{4,8}$.

Using Eqs. (1) and (2) we define the power efficiency factor $P_{E F F}$ of the algorithm for idle nodes by

$$
\begin{aligned}
& P_{E F F}=\frac{E_{1}-E_{2}}{E_{1}}=\frac{\left(P_{L}-P_{S}\right) * T_{S}}{P_{L}^{*} T_{W}} \\
& K_{D E V}=\frac{\left(P_{L}-P_{S}\right)}{P_{L}}
\end{aligned}
$$

The constant value $K_{D E V}$ depends on the hardware specifications of the wireless card and bounds the maximum value of the power efficiency factor. The maximum number of retransmissions required for a sleeping node to receive a packet is defined by

$$
R=T_{w} / T_{O}, \text { where } T_{L}>T_{O}
$$

Also we define the ratio $D=T_{S} / T_{o}$

Using Eqs. (3), (4), (5) and (6) the power efficiency is found to be,

$$
P_{E F F}=K_{D E V} * \frac{D}{R}, \text { where } R \in N^{+} \text {and } R>1
$$

A guideline to select adequate parameters for the algorithm is the following; $T_{S}$ must have a small value to reduce latency. A positive integer is selected for the number of retransmissions $R$ in order to achieve the desirable energy efficiency. The minimum value of $T_{L}$ depends on the MAC layer specifications. Also $T_{O}$ must be lower than $T_{L}$ by an amount that reflects possible transmission delays caused by the 
driver, operating system, packet transmission delay or the MAC congestion avoidance mechanism. A set of values that has been used in the implementation and in the simulations of Section 5 is the following: $T_{L}=69 \mathrm{~ms}, T_{S W}=1 \mathrm{~ms}, T_{S}=290 \mathrm{~ms}, T_{O}=60 \mathrm{~ms}$ and $R=6$. This set in case of the Orinoco wireless card $\left(\mathrm{P}_{\mathrm{TX}}=1408 \mathrm{~mW}, \mathrm{P}_{\mathrm{RX}}=914 \mathrm{~mW} \mathrm{P}_{\mathrm{IDLE}}=785 \mathrm{~mW}\right.$ $P_{\text {SLEEP }}=65 \mathrm{~mW}$ ) can achieve $P E F F=0.73$.

\section{POWER AWARE ROUTING}

There have been several research efforts regarding power aware routing algorithms. These algorithms must select the best path to minimize the total power needed to route packets on the network and maximize the lifetime of all nodes. Minimum cost battery routing $\mathrm{MCBR}^{9}$ proposes the remaining battery capacity of the nodes as a metric. Min-max battery routing MMBR ${ }^{10}$ defines as a cost metric of a route the maximum battery cost value of the nodes that constitute the path. Although the previous algorithms reduce network consumption and increase node lifetime, or both, in networks where the wireless channel is characterized by multi-path propagation it is observed that some links may experience an increased packet error rate. These unstable links can decrease the network performance significantly ${ }^{7}$ due to packet losses and the initiation of the route discovery mechanism.

Efforts towards this direction have been also made to add criteria based on a combination of shortest-path, link quality, or least congested paths, that is, network load. Link quality estimations can be based on either the signal-to-noise ratio or the expected transmission count (ETX) metric $^{12}$. The results Couto ${ }^{12}$ and Draves ${ }^{13}$ show that with stationary nodes the ETX metric significantly outperforms shortest path routing; also network-load and packet delay metrics perform poorly because they are load-sensitive and hence suffer from self-interference. However, Draves ${ }^{13}$ concludes that in a mobile scenario shortest-path routing performs better, compared to ETX, because it reacts more quickly to fast topology change. Therefore the path length is also considered in the metric for our route selection.

In this work we present a route selection algorithm that combines link stability metric with the battery level of the nodes and the route length. When the nodes have battery levels above some threshold and the links are stable, shortest path routing is performed. In case of link failures stable links are preferred and as the battery level of nodes is decreased a combination of the above metrics is encountered in route selection. Following the methodology proposed by Singh ${ }^{9}$ we define the path cost $C\left(n_{0}, n_{k}\right)$ of a path from a source node $n_{0}$ to a destination node $n_{k}$ by:

$$
C\left(n_{0}, n_{k}\right)=\sum_{i=1}^{k} z\left(n_{i-1}, n_{i}\right)
$$


where $z\left(n_{i-1}, n_{i}\right)$ is the cost of the link from node $n_{i-1}$ to node $n_{i}$. The path cost $C$ depends on the path length of the route and the cost of the links that compose the route. The link cost is defined as a function of the energy cost Zen and the stability cost Zst of the link from node $n_{i-1}$ to node $n_{i}$ by:

$$
z\left(n_{i-1}, n_{i}\right)=f\left(\operatorname{Zen}\left(n_{i}\right), Z s t\left(n_{i}\right)\right)=\operatorname{Zen}\left(n_{i}\right) * Z s t\left(n_{i}\right)
$$

The multiplication of the above metrics increases the cost of nodes that have unstable links and low energy levels. As a result longer hop paths are preferred and packets are routed over these nodes. The energy cost metric of a node is defined as:

$$
\operatorname{Zen}\left(n_{i}\right)=1+E F^{*} g\left(n_{i}\right)
$$

where $g\left(n_{i}\right)$ is the normalized energy consumed by the node, $g\left(n_{i}\right)=\left(E_{\text {INITILL }}-E\left(n_{i}\right)\right) / E_{\text {INITIAL }}, E F$ is the energy cost factor which bounds the maximum value of the energy cost, $\mathrm{E}_{\text {INITIAL }}$ is the initial and $E\left(n_{i}\right)$ the current residual energy level of the node. The stability cost metric is defined as:

$$
Z s t\left(n_{i}\right)=1+\frac{1+S F}{\text { stability }\left(n_{i}\right)}
$$

where $\operatorname{stability}\left(n_{i}\right)$ is the stability metric of that node and $S F$ a stability cost factor which bounds the maximum value of the stability cost. An optimal assignment of the $\mathrm{EF}$ and $\mathrm{SF}$ factors is required for meeting the network communication constraints. Increasing EF a priority is given to power aware routing, whereas increasing SF more stable links are preferred.

The stability metric was first proposed by $\mathrm{Hu}^{11}$ in order to estimate a dynamic link timeout cache policy, the Link-Max-Life (LMF) link cache. When a node is added in the cache it has an initial link stability value (SINITIAL). When a link from the route cache is used in routing a packet originated by that node, the stability metric of the two end point nodes is additively increased by a stability increase factor $S I N C F$, $\operatorname{stability}\left(n_{i}\right)=\operatorname{stability}\left(n_{i}\right)+\operatorname{SEDCF}$.

Upon a link error, the source node will receive a route error packet containing the broken-link. In this case the stability metric of the two end point nodes is multiplicatively decreased by a stability decrease factor 1/SDECF (where $S D E C F \geq 2$ ), stability $\left(n_{i}\right)=\operatorname{stability}\left(n_{i}\right) / S E D C F$.

In any case the stability metric is bounded in a set [2, MAX_STAB]. Using Eqs. (10), (11) and (12) the path cost is equal to: 


$$
C\left(n_{0}, n_{k}\right)=\sum_{i=1}^{k}\left(1+E F^{*} g\left(n_{i}\right)\right) *\left(1+\frac{1+S F}{\text { stability }\left(n_{i}\right)}\right)
$$

In the presence of unstable links in the network, nodes that route packets capture the received route error messages and update the stability metric of the nodes in the network. Each node maintains in the route cache a local view of the stability metric of links in the network. As a result, nodes that experience a high link loss ratio have decreased values of stability. Using Eq. (12) source nodes in the network route packets avoiding the use of unstable links and nodes with low energy, increasing the network lifetime and communication robustness. In Table 1, a comparison is presented between different wireless cards: the Agere Orinoco Gold and the Cisco Aironet PC4800B. In the case of the Orinoco wireless card, the increased value of $K_{D E V}$ implies that the LPM algorithm is more effective, as the $P_{E F F}$ can attain higher maximum values. A set of values that has been experimentally evaluated in the deployed wireless ad hoc network is the following: $E F=1, S F=2$, $S D E C F=2, S I N C F=2, S I N I T I A L=25, M A X \_S T A B=300$.

Table 1. Measured power consumption of IEEE 802.11 wireless cards

\begin{tabular}{|c|c|c|c|c|c|}
\hline Minivers & 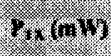 & $\mathrm{R}, \mathrm{nW}$ & Printin & $(x-9,(n) 1)$ & nen. \\
\hline Aroold & 1729 & 1380 & 1300 & 320 & 0.7598 \\
\hline Orhoke & 1408 & 914 & 785 & 65 & 0,9171 \\
\hline
\end{tabular}

\section{SIMULATION STUDY}

In this section we evaluate the performance of the proposed LPM and PAR algorithms and their effect in communication efficiency with simulations using the ns-2 network simulator. The network topology consists of 50 nodes randomly distributed in a $1000 \mathrm{mx} 1000 \mathrm{~m}$ square area. The results are average values for a number of simulations in which the nodes are either static or have a random way point movement with relatively low mean speeds varying from 0 to $5 \mathrm{~m} / \mathrm{sec}$. 


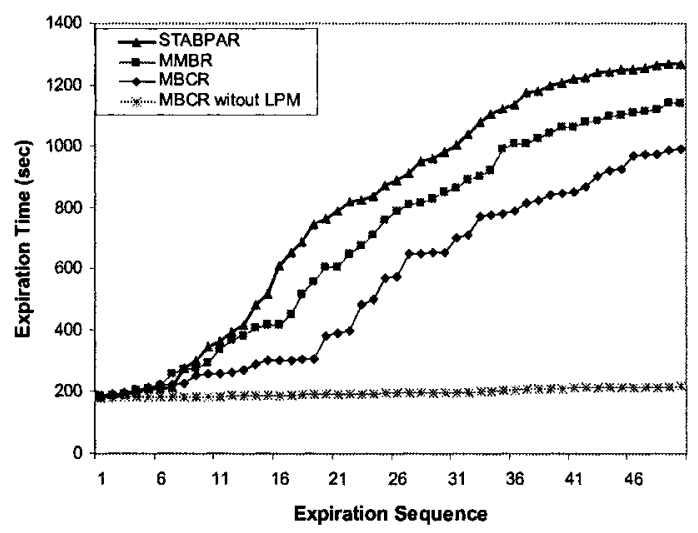

Figure 3. Expiration time vs. expiration sequence

In Fig. 3 we present the expiration time of each node in the network (i.e., the time when a node exhausts its battery) for four different cases: when the LPM is not used and the routing strategy is MMBR, when LPM is used with MBCR and MMBR routing and when LPM is used with the proposed power aware routing (STABPAR). The presented simulation results are in a network topology with 50 nodes and 5 simultaneous CBR traffic flows of $80 \mathrm{Kbps}$. As we can observe, the combination of the proposed power aware routing and the LPM algorithm can achieve a significant increase in the lifetime of nodes. In this case energy efficient routes that experience a lower link-loss ratio are preferred for routing packets, as a result there is a significant decrease in route errors and route discovery operations that waste energy.

\section{CONCLUSIONS}

In this paper we have presented a Low Power Mode (LPM) algorithm and a Power Aware Routing (PAR) strategy to reduce network consumption and increase node lifetime in an efficient way. Although this work is focused on 802.11 and DSR it can be applied to other MAC and routing protocols, as well. The LPM algorithm can reduce the energy consumption of the wireless card up to $70 \%$, while the network performance is not affected significantly. The PAR strategy proposes a new metric for route selection which combines the link stability and the node battery levels. As a result, energy efficient stable links are preferred for routing packets and, thus, node lifetime and network robustness is increased. The combination of the proposed LPM and PAR algorithms 
can lead to powerful energy efficient solutions for ad-hoc wireless networks.

During some preliminary experiments in real wireless networks, deployed with embedded sensor nodes and mobile PCs as central units, the network lifetime was increased significantly and the network performance was improved. The experimental evaluation of the stability metric in the presence of high attenuation and interference show that more stable routes were selected increasing the throughput in multi-hop TCP connections between the sensor nodes and the central stations, compared to shortest-path routing. As part of our on-going work we experimentally evaluate the PAR and LPM algorithms and several aspects of the protocol stack, such as TCP performance and tuning, in a wireless network with a large number of nodes.

\section{REFERENCES}

1. “LOCCATEC: Low Cost Catastrophic Event Capturing," European Research Project IST-2000-29401, www.loccatec.org

2. N. Pogkas et al. "System for acquiring and surveying data following catastrophic events, with scope of facilitating eventual aid or intervention," European Patent No. 03425667.7 .

3. D. B. Johnson, D. A. Maltz, and J. Broch. "The Dynamic Source Routing Protocol for Mobile Ad Hoc Networks," Internet Draft, MANET Working Group, draft-ietfmanet-dsr-09.txt, April 2003.

4. R. Min and A. Chandrakasan, "Top 5 Myths about the Energy Consumption of Wireless Communication," IEEE Mobihoc 2002.

5. B. Chen, K. Jamieson, H. Balakrishnan, and R.Morris, "Span: An Energy- Efficient Coordination Algorithm for Topology Maintenance in Ad Hoc Wireless Networks," Proc. of the International Conference on Mobile Computing and Networking, pp. 8596, 2001

6. R. Zheng, J. C. Hou and L. Sha, "Asynchronous Wakeup for Ad Hoc Networks," MobiHoc 2003.

7. K. Benekos, N. Pogkas, G. Kalivas, G. Papadopoulos, "TCP Performance Measurements in IEEE 802.11-based Wireless LANs," IEEE MELECON, May 2004.

8. J. C Cano., P. Manzoni, "A Performance Comparison of Energy Consumption for Mobile Ad Hoc Networks Routing Protocols," IEEE ACM MASCOTS, 2000.

9. S. Singh, M. Woo, C. S. Raghavendra, "Power-Aware Routing in Mobile Ad Hoc Networks," Proc. Mobicom, Oct. 1998.

10. C. K. Toh "Maximum Battery Life Routing to Support Ubiquitous Mobile Computing in Wireless Ad Hoc Networks," IEEE Communications Magazine, June 2001, pp 138-147.

11. Y. C. Hu and D. B. Johnson., "Caching Strategies in On-Demand Routing Protocols for Wireless Ad Hoc Networks," Proc. ACM International Conference on Mobile Computing and Networking, August 2000.

12. D. Couto, D. Aguayo, J. Bicket, and R. Morris, "A High-Throughput Path Metric for Multi-Hop Wireless Routing," MobiCom 2003, September 2003.

13. R. Draves, J. Padhye, and B. Zill, "Comparison of Routing Metrics for Static MultiHop Wireless Networks," ACM SIGCOMM, August 2004. 